\title{
The Development of Cytological Method for the Demonstration of Larval Polytene Chromosomes of Aedes Aegypti.
}

\author{
Aju-Ameh, Onyawoibi Celina and Mafuyai, Hayward Babale \\ Department of Zoology, University of Jos, Jos, Plateau State, Nigeria.
}

\begin{abstract}
Aedes aegyti (Diptera: Culidae) the yellow fever vector was successfully colonized under laboratory conditions. Aedes aegyti larvae were collected from different sites in the wild and brought into the laboratory and the salivary gland polytene chromosome squashes were examined. The phenomenon of staggered hatching was observed, and two morphotypes of Aedes aegypti were encountered from the wild, thus it became necessary to investigate the polytene chromosomes to discover in any revelations of differences in their chromosomal sequences that might lend themselves to species identifications.
\end{abstract}

Keywords:Aedes aegypti morphotypes, Cytological technique, Larval instar, Polytene chromosomes, Salivary gland.

\section{Introduction}

Mosquitoes are reported to have a world wide distribution occurring in both the tropical and temperature regions. They are reputed to be the most prominent of all the numerous kinds of bloodsucking arthropods that annoy man, other mammals and birds. Their attacks are not confined to warm blooded animals as there are records of mosquitoes that fed on fishes, reptiles and amphibians, and in the process transmitting pathogens. The number of species is legion and great swarms are often produced from practically all sorts of still water, fresh and brackish, fowl or clear water, water in tin cans, car and Aeroplane tyres, hoof prints, tree holes, water deposits in leaf cups, the margins of Streams, Rivers, Lakes and water impoundment (Service .M.W 1980,Robert et al 1977; Gillet, J.D 1971 and Bates .M 1949).Proper identification is vital in studying and combating mosquito-borne diseases. The keys used for the identification of the mosquitoes in this project work were adapted from (Gilles and De Millo 1968; Gilles and Coetzee 1987) were also used. The classification of mosquitoes by the use of external morphological criteria is satisfactory only to a certain degree, but in some disease situations conventional morphological characteristics are of limited use. This is usually in situations involving complexes of species and the success in disease control is often limited. Zahar 1993 and White et al 1975 have reviewed concepts for discriminating species and subspecies. According to their report taxonomist routinely examine the senilae on antennae and perform cross-mating experiments, polytene chromosomes, and comparisons of electrophoretic patterns of enzyme systems (Colluzi 1970, Coluzzi et al 1985). While the polytene chromosomes of Aedes aegypti have been difficult to obtain a lot of work has been done in the case of Anopheline mosquitoes. This research work is planned as continuing effort to obtain polytene chromosomes of Aedes aegypti. Any success achieved will open up new frontiers of the basic knowledge of the polytene chromosomes of Aedes aegypti mosquitoes; leading to a better appreciation of their identity and disease involvement.

1.1 Mosquito genetics and cytogenetics: Genetic work on mosquitoes really began in an organised way with the publication of the monograph by Kitzmiller $(1967,1953)$ on "Mosquito genetics and cytogenetics" Until that time, genetic studies on Mosquitoes had been desultory and incidental to other research objectives. The development of insecticide resistance in the decade between 1945 and 1955 acted as a major stimulus for mosquito cytogenetic studies, indeed, resistance brought about an awareness of genetics on the part of entomologists in general (Busvine 1957; Brown 1973). Aedes aegypti is one of the best known insects from the standpoint of both basic and applied science. Because of its medical importance a vast body of literature has accumulated concerning its systematic, distribution, bionomics, disease relationships and control. Its occurrence in domesticated habitats in much of the tropical world makes it a familiar denizen of the household. It is readily adaptable to laboratory culture and has a high reproductive potential, short generation span, the possibility of long-term storage of eggs up to one year, low chromosome number three pairs (2n-6) and a rich store of genetic variability (brown et al1995). In addition, the great accumulation of information on physiology and bionomics of this species provided an ideal foundation for the initiation of genetic studies. Aedes aegypti is indeed a good candidate for most sorts of genetic investigations. The amount and range of information accumulated is evident in the 739 pages and 2387 references in the book by Sir, Richard Christopher's Aedes aegypti, the yellow fever mosquitoes, it's life history, 
bionomics and structure, but there has not been much success in the spreading of polytene chromosomes in culicine mosquitoes. So far very little is known about the chromosomal banding patterns.

1.2 Polytene chromosomes: Polytene Chromosomes according to the dictionary of genetics are giant cable like chromosomes consisting of many identical chromosomes closely associated along their length, with their chromosomes in register. Balbiani first described polytene chromosomes in 1881 , but it was not until about fifty years later that more detailed study revealed their true significance. Polytene chromosomes arise from normal somatic chromosomes which undergo several rounds of DNA replication (repeated chromosome duplication) with accompanying nuclear cell division. The cells are therefore polyploid but the chromatids remain laterally associated to produce the polytene type of organisation. They are very good and have been used for clarifying species complexes. Aedes aegypti is said to be polymorphic and at least three varietal forms have been recognized. The identification of these populations based on morphological, physiological and behavioural differences is an indication of differences in genetic load (Spielman and Kitzmiller1967). The difference in genetic load is due to variations in chromosome morphology. Such variations could include several inversions, as a result of which some other inversion effects become suppressed, leading to occasional gene length among others (Gale and Crampton 1989).On the whole culicine complexes appear to be rarer and identification has been based on morphological taxonomy.

\section{Materials And Methods}

Aedes larvae were collected from the rock pools around the Remedial and Natural Science buildings of the university of Jos and brought into the laboratory. The larvae were fed on dry yeast powder. A small, but adequate quantity of yeast was used to feed the larvae twice daily in the morning and evening. The containers were checked daily for pupation and pupae were collected using a wide mouthed pipette. They were then transferred into a small dish and placed in an emergence cage. As they emerge from the pupal cases they are trapped in the new cage. The adult mosquito species were bred in the laboratory using a mosquito cage cubit netting cages of dimensions $15-30 \mathrm{~cm}$. They were maintained under constant laboratory conditions at a temperature of $25^{\circ} \mathrm{C}$ with a 12 hour photoperiod and Relative humidity of between $70-80 \%$. Adults were at a density of 50-300 per cage. The adults were fed on a $10 \% \mathrm{w} / \mathrm{v}$ sucrose or sugar solution given in a cotton wool pad on top of the cage. The cotton wool was soaked slightly to prevent the sugar solution from dripping into the bottom of the cage and killing the mosquitoes. The cotton wool pad was further covered with a plastic lid to prevent evaporation and contamination by house flies. The adults were blood fed at least once a week, for a period of 15-20 minutes. A small dish of water lined with filter paper was provided for oviposition. The eggs hatched into the larvae, which are transferred to a large plastic bowl of water at a density of 0.5 larvae per square centimeter of water surface. The method by Hunt 1973 as reported by White et al(1975) was adopted. The technique employed used carnoy's fixative as preservative for whole larva. After preservation at room temperature for 24 hours, the material was held at about $4^{0} \mathrm{C}$ in a refrigerator.

2.1 Larval Dissection: The larva was placed on a slide with a droplet of $50 \%$ propionic acid for 30-60 seconds. The larval abdomen was then cut off and discarded. The thorax was then slit open dorsally by running a needle over it to sever the layers between. Gentle traction on the head usually pulls the glands free. The salivary glands were then macerated, clearing excess tissue from the chromosomes. A droplet of diluted aceto-lactic orcein $(2 \%$ aceto-lactic orcein diluted 1:10 with $50 \%$ propionic acid) was then added and mixed with the tissues. Excess was blotted away after half a minute and the tissues washed with successive droplets of 50\% propionic acid. The preparation was then covered with a clean cover slip and squashed. By using a flat bottom pen used for the tapping, while checking for adequate spreading of the chromosomes. The squashed preparation were made permanent (fixation and staining) as soon as the chromosomes were judged to be adequately spread. They were sealed using rubber solution all around the edge of the cover slip and kept in the refrigerator.

\section{Results}

Aedes aegypti was common from the months of June to August and had a peak period in July and August. There was a decrease in their population from September through December around the University (Bauchi Road Campus \} environment. A total of one hundred and five larvae were collected from the wild and reared up to the adult stage. A total of four hundred and seventy-seven (477) eggs 
were laid by the single parent specie picked up from the mixed colony cage to a single parent cage. A chi-square test showed that there was no significant difference ( $p>0.05 \%$ ) between the number of eggs laid and the number expected. Three surfaces were provided for the oviposition activity of the female Aedes aedvpti. Amongst the test surfaces were free water in a bowl, mud surface and wet filter paper. A clear preference was shown for the free water surface. The eggs did not hatch readily as expected (Table 2).Larval development from one instar to the other, and to the pupa and adult stages, within any one twenty-four hours ranged from zero (none) to five (5) during the period of study. The males emerged before the females. Chromosomal preparations of the second and third larval instars showed nuclei not spreading (plates 2 and 3).A fourth instar larvae of Aedes aegvpti was then dissected and the nuclei stained faintly spreading more. The structure deteriorated within the first twenty-four hours of its preparation, and it was attributed to the fact that the cover slip was wrongly placed as such it let in air.

\section{Discussion}

The majority of studies carried out on mosquitoes are in relation to the diseases they transmit and Aedes aegypti is the principal yellow fever vector (service 1980, Miller et al 1989). The well documented habit of staggered hatching (TABLE 1) was recorded for Aedes aegypti Mafuyai (1984). This is a survival strategy for these species, as they breed in temporary pools that are subject to drying. However the small number of eggs laid is correlated to the amount of blood ingested (Clements 1963).Attempts to get most of the eggs to hatch failed(TABLE 2) and this could be attributed to several reasons amongst which are, chemical composition of water, an inter play of other environmental factors (for example temperature, relative humidity, and speed, rainfall etc), which cannot be controlled and an arrested development in egg stage - Diapuase (Clements 1963, Kettle 1984, Service 1980).Aedes aegypti laid eggs just above the water level in a small bowl in preference to the other surfaces provided. Mafuyai (1984) reported that the selection of oviposition sites by mosquito species is usually governed by the suitability of the physical properties of the substrate, the amount of moisture, the degree of reflectance from it and the texture, colour and other chemical factors of a water body. The successful breeding of the larvae of the species under the laboratory condition provided evidence of their high survival and adaptive powers. Salivary gland dissections proved very difficult at the beginning for species. This was due largely to inexperience and the very sensitive and delicate steps 'involved in the dissection and preparations of the chromosome squashes. According to White et al (1975) attempts at spreading the polytene chromosmeso Aedes aegypti have not been successful. Our report here confirms this difficulty after we had used the second and third instars and their nuclei failed to spread (Plates 2\&3). A fourth laval instar preparation was made and a significant spread of the nuclei observed. The seasonal change from the wet season to the dry season resulted in a drop in adult population. Seasonal variations revealed Aedes aegypti had a peak in July and August. The erratic overflowing of the breeding sites due to sudden and rapid flushes of rains made most breeding sites unproductive for the larvae. During the heavy rains the larval population was usually flushed away. A 2 x 2 factorial design was used to investigate interaction between the seasons (months) and the sites (habitats) and an analysis of variance revealed a highly significant interaction between seasons and sites at $(\mathrm{P}<0$. 05) level of significance. A least significance difference test (LSD) showed that the ground rain water habitat was significantly different from the other two habitats (stream edge and artificial container) with the highest number of larval collections. There were several limitations that militated against the successful and detailed study intended for the purpose of this research work. Some of the limiting factors are as follows:(i) the fact that the insectary is only partially functional. This made laboratory colonization of the species very difficult; as the weather elements could not be manipulated to our advantage.(ii)Lack of a good photomicrograph system for immediate snap shots and successful processing and production of acceptable photomicrographs, (iii) Lack of Normaski microscopy. Structures in the polytene nucleus such as the nucleous and the Balbiani rings may not be readily apparent in preparations which have been fixed, stained or dehydrated. Macgregor and varley (1988) advocate the use of phase contrast or Normarski interference microscope for visualization of micromorphological features of the polytene chromosome. (iv)Incessant power supply, made working with both the dissecting and light microscope very frustrating. Although a generator was provided by the supervisor, the freezers and refrigerator with the fixed specimens had insufficient hours of power supply for the right temperature for such specimens. This greatly altered the chromosomes being prepared. Apart from the above reasons for chromosomal alterations, another source of variation in chromosome 
preparations as stated by Green (1971) is the result of mechanical stresses of the squashing operation. This usually lead to various degrees of stretching, which in turn may lead to some difficulty in using the maps. Also there are suggestions as to the possibility of sibling speciation in Aedes aegypti the yellow fever vector. During the cause of this study, two forms of Aedes aegvpti were encountered. The dark-type-form and the light-type-form, from their conventional morpological features. The wings of one being darker than that of the other. Man is said to represent an important factor in environmental changes and heterogeneities and as such acts as a powerful evolutional force in the speciation processes. Coluzzi et al (1979) started that Man-influenced genetical variations in mosquito vector systems are in many cases epidemiologically crucial. To ignore this genetical heterogeneity can mean, and has meant, incorrect evaluation of the basic parameter employed to assess vectorial capacity and its reduction due to vector control measures. Furthermore, the knowledge of the genetical factors involved in the evolution of mosquito adaption to man and his environment has more than theoretical interest. It has practical significance for planning of control strategies (Moriss et al,1989). The importance of studying Aedes aegypti is still relevant today. Polytene chromosomes provide the distinction of the sibling species and thus aid the medical entomologist in the control and eradication of these disease vectors.

\section{Conclusion}

This study has attempted to improve the method/technique for preparing polytene chromosomes of Aedes aeavpti. A promising lead in the discovery of the fourth larval instars for good polytene chromosome preparations has been made. However, other tissues such as the malphigian tubules, ovarian nurse cells, in the adults require further investigation and the salivary gland techniques for the larvae need to be reviewed. These objectives may only be realized in longer span time studies in well equipped laboratories and could not be realized within the context of a short study such as this.

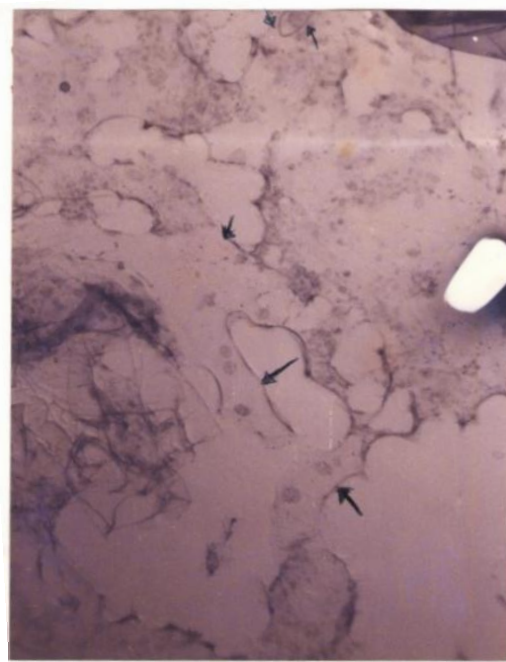

Plate 2: Second instar larval salivary gland Preparation Aedes aegypti X40

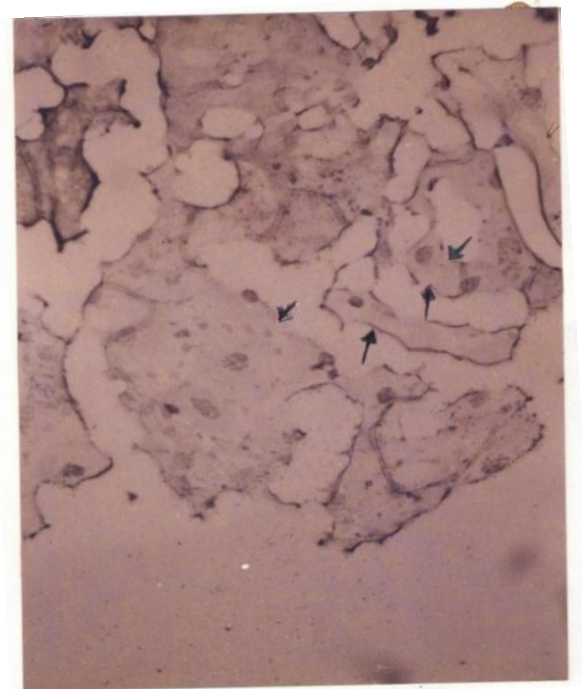

Plate 3: Third Larval salivary gland chromosome preparation Aedes aegypti X40

TABLE:1, Submergence and Hatching Of Aedes Aegypti Eggs

\begin{tabular}{|c|c|c|c|c|c|c|c|}
\hline Months & $\begin{array}{l}\text { Date } \\
\text { Introduced }\end{array}$ & $\begin{array}{l}\text { Date of eggs } \\
\text { count First }\end{array}$ & First & Second & Third & Fourth & Fifth \\
\hline Sept & $12-08-97$ & $\begin{array}{l}15-08-9721- \\
82\end{array}$ & $\begin{array}{l}9-97 \\
40\end{array}$ & $\begin{array}{c}27-9-97 \\
12\end{array}$ & $\begin{array}{c}30-9-97 \\
1\end{array}$ & $\begin{array}{c}4-10-97 \\
0\end{array}$ & $\begin{array}{c}10-9-97 \\
0\end{array}$ \\
\hline
\end{tabular}

Eggs were laid on only the free water surface $94.99 \mathrm{~cm}^{2}$

No Eggs were found on the MUD surface and filter paper.

Null hypothesis : Observed data does not deviate significantly from the expected one 
$x^{2}$-test our observed are as follows $91^{(\mathrm{a})} 82^{(\mathrm{b})} 82^{(\mathrm{c})} 63^{(\mathrm{d})} 93^{(\mathrm{e})} 66^{(\mathrm{f})}$ which were used to get the expected values by preparing a 2 by 3 contingency table. Our $\mathrm{X}^{2}$ for $\mathrm{P}=0.05$ with two degrees of freedom is 5.991Therefore, since the calculated $X^{2}$ value is 1.2169 and tabulated is 5.991 we retain our null hypothesis.

TABLE 2 Submergence and Hatching of Aedes Aegypti Eggs Monthnumber of eggs SUBMERGENCE counted

\begin{tabular}{|c|c|c|c|c|c|c|c|}
\hline & & $1^{\mathrm{st}}$ & $2^{\text {nd }}$ & $3^{\mathrm{rd}}$ & $4^{\text {th }}$ & $5^{\text {th }}$ & Total hatched \\
\hline July & 91 & 0 & 1 & 0 & 0 & 0 & 1 \\
\hline August & 82 & 32 & 10 & 2 & 0 & 0 & 44 \\
\hline September & 82 & 40 & 12 & 1 & 0 & 0 & 53 \\
\hline October & 63 & 0 & 1 & 0 & 0 & 0 & 1 \\
\hline November & 93 & 0 & 0 & 0 & 0 & 0 & 0 \\
\hline December & 66 & 0 & 0 & 0 & 0 & 0 & 0 \\
\hline Totals & 477 & 72 & 24 & 3 & 0 & 0 & 99 \\
\hline
\end{tabular}

$\mathrm{X}^{2}=1.2169 \mathrm{P}>0.05 \%$

\section{Acknowledgement}

The author is grateful to her supervisor Professor H.B.Mafuyai for facilities provided especially his personal generator few years back for the progress of the work.

\section{Reference}

[1]. Service M.W. (1980\} A. Guide to medical entomology Macmillan International College editions (Mice) 22-70.

[2]. Roberts,D.W. and Strand.M.A.(1977) Pathogens of Medically Important Arthropods,Supplement no.1Bull.Wld Health org., 55;419pp

[3]. Gillet,J.D(1971) Mosquitoes, weidnenfeld\& Nicolson,London,274pp

[4]. Bates .M (1949) The Natural History of Mosquitoes. Haper and Row 112-116.The Macmillianco.NewYork,republished by HarperTouch books, 1965 .

[5]. Gilles and De Meillon (1968) Key to Female Anopheles of Nothern Nigerian. I

[6]. Gilles, M.T. and Coetzee M. (1987) A. Supplement to the Anophelinae of Africa South of the Sahara, (Afro tropical Region) Publ. S. Afri. INST. Med. Res. No. 55: 3, 1936.

[7]. Zahar A.R. (1 993) Review of Advances made in the recognition of members of the Anophelesgambiae complex and their Bionomics in the Afrotropicalregion:In: Coetzee M(ed) Entomologist Extraordinary (1993). 64-77.

[8]. White G.B., Coluzzi M., and Zahar A.R. (1975) Review of Cytogenetic studies on Anopheline vectors of Malaria, Bull of W.H.O. (1975) 1-15.

[9]. Coluzzi M. Sabatini A., Petrarca V., and Di Deco M.A. (1979) Chromosomal differentiation and adaptation to human environments in the Anopheles qambiae complex. Trans. Roy. Soc. Med. Hyg. Vol. 73 No. 5.

[10]. Coluzzi M. Petrarca V., Di Deco M.A. (1985) Chromosomal inversion intergradation and Incipient Speciation in Anopheles qambiae Bull Zool 52: 45-63.

[11]. Kitzmiller J.B. (1967) Mosquito Cytogenetics |n: Wright J.W, and Pal R, (eds) Genetics of Insect vectors of disease. Elservier Publishing Company Amsterdam 133-144.

[12]. Kitzmiller J. B. 1953 Mosquito genetics and cytogenetics.Separata da Rev. Bras. deMalariol. e D. Trop. 5, $285 \pm 359$.

[13]. Busvine, J.R, (1957) Insecticides resistance strains of Insects of Public Health Importance. Trans. Roy. Soc. Trop. Med. Hvg. 51, 11-31.

[14]. Brown A.W.A. and Pal R. (1973) Insecticide resistance in arthropods, W.H.O. Monngr. Ser. 38.541

[15]. Brown S.E, Menninger J., Dillipantonio M. Beaty. B.J., Ward D.C, Knudson D.L. (1 995) Toward the physical map of Aedesaegypti Insect-Mol-Biol 1995 Aug:4 (3) 161-7

[16]. Balbini,E.G.(1881).Sur la structure du noyau des celluclessalivaires chez les larves de chromosomes.zool.Anz.4,637-641,662-666.In Macgregor H. and Varley J. (1988) Working with animal chromosones. John Wiley and Sons 73-110, 117-143.

[17]. Spielman, A and JG Kitzmiller. 1967. The genetics of populations of medically important arthropods. in JR Wright and R. Pal (eds), Genetics of Insect Vectors of Disease. Elsevier, Amsterdam pp 459-485.

[18]. Hunt,R.H (1973) A cytological technique for Studying of Anopheles gambiaecomplex:In White G.B., Coluzzi M., and Zahar A.R. (1975) Review of Cytogenetic studies on Anopheline vectors of Malaria, Bull of W.H.O. (1975) 1-15.

[19]. Gale K., and Crampton J. (1989) Theribosomaf genes of the Mosquito, Aedesaegypti. Eur-J-Biochem. 1989 Nov. 6: 185 (2): 31 1-7.

[20]. Miller B.R. Monath T.P., Tabachnick W.J., Ezike V.I. (1989) Epidemic Yellow fever caused by an incompetent mosquito vector.Trop-Med - Parasitol. 1989 Dec. 0 (4): 396-9.

[21]. ]Mafuyai B.H. (1984) The Ovipositional preferences of some mosquitoe species under laboratory conditions B.Sc. Thesis, University of Jos, Plateau State, Nigeria (unpublished).

[22]. Clements A.N. (1963) The Physiology of Mosquitoes, A Pergamon Press Book.TheMacmillan Company, New York. 1963, 1-30, 128-146; 164-188 and 220-261.

[23]. Kettle D,S. (1984). Medical and Veterinary Entomology Crom Helm London and Sidney.99-133.

[24]. Macgregor H. and Varley J. (1988) Working with animal chromosones. John Wiley and Sons 73-110, 117-143.

[25]. Green C.A. (1971) Cytological Maps for the practical identification of the females of the three fresh water species of the Anopheles gambiae complex. Annals of Trap.Med. and Para Vol. 66(i) 143-147.

[26]. ColuzziM.,Sabatini A.,Petrarca v., and Di Deco M.A.(1979) Chromosomal Diferentiationadaptationto human environments in Anopheles gambiae complex.Trans.Roy.Soc.Med.Hgy.Vol.73 No.5.

[27]. Morris A.C., Eggleston P., and Crampton J.M. (1989). Genetic transformation of the mosquito Aedesaeqypti by micro-injection of DMA Med. Vet. Entomol.1989 Jan., 3 (7)1-7. 